\title{
Innovative Technologies in Tailoring Designer Food and Personalized Nutrition
}

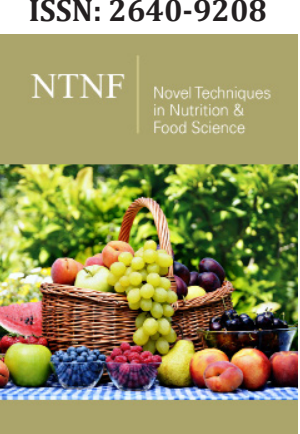

*1Corresponding author: Kumar GT, Department of Agriculture and Food Engineering, IIT Kharagpur, India

Submission: 眥 July 02, 2019

Published: 酱July 23, 2019

Volume 4 - Issue 2

How to cite this article: Baishakhi D, Koushik B, Kumar GT. Innovative Technologies in Tailoring Designer Food and Personalized Nutrition. Nov Tech Nutri Food Sci. 4(2).NTNF.000582.2019. DOI: 10.31031/NTNF.2019.04.000582

Copyright@ Kumar GT, This article is distributed under the terms of the Creative Commons Attribution 4.0 International License, which permits unrestricted use and redistribution provided that the original author and source are credited.

\author{
Baishakhi D ${ }^{1}$, Koushik B $^{2}$ and Kumar GT ${ }^{3 *}$ \\ ${ }^{1}$ SSS Indira college of Pharmacy, Maharashtra, India \\ ${ }^{2}$ Advanced Technology Development Centre, IIT Kharagpur, India \\ ${ }^{3}$ Department of Agriculture and Food Engineering, Kharagpur, India
}

\begin{abstract}
This concise review has made a discussion on different thermal and non-thermal techniques associated with novel food processing technologies. Further a focus has been made on 3D food printing for mass customized diet and personalized nutrition.
\end{abstract}

Keywords: Food processing; 3D food printing; Mass customized diet; Personalized nutrition;

Thermal and non-thermal techniques

Introduction

Dietary and lifestyle interventions have become a part of therapeutic regimen. The enhanced cognizance amongst the consumers obviously increased competition and dynamism amongst food industries. Today's well-informed consumer world demand low caloric diversified food products, both nutritional and health benefits with value addition, convenience, extended shelf life, cost effectivity and other environmental credentials $[1,2]$. With the understanding of human genome and implementation of integrated "omics technologies" personalizing nutrition and medicine are the new frontiers with several challenges [3]. Personalized nutrition and thus customized food products encompass a convergence of the advancements of science and technologies in varied disciplines and its popularity amongst consumer world is observed with the hypes of "free diets" (calorie free, gluten free, lactose free etc.) [4]. Customized foods are tailor made or designer foods that are developed to meet the specific needs of each person depending on their taste, physical and health status. Mass customized food items though fulfill the needs of wider consumer community however its further development depends on the in-depth understandings of human genome and subjects of nutrigenetics (interactions between food and human body) and nutrigenomics (the effect of diet on gene activity). Then designer or customized food will not only satisfy the health needs but also will serve the purpose of adjunct therapy in many chronic ailments viz. metabolic syndrome, neurodegenerative disorders, cardiovascular diseases; deficiency syndromes, blood and bone health etc. [4].

Development of new designer food products necessitates a thorough understanding of the associated technological know-how, company strategy, brand portfolio, marketization and parallel competitions, and finally the latent needs of the end users-the consumers. The design thinking approach with value addition at different steps aim to make products much more end-user oriented. The fork to farm projects also tries to maximize the benefits of the end-users. The design thinking and the designers handle the design challenges holistically; devising and designing the equipment's and processes of the product development, coordinating amongst disciplinary experts and integrating amongst different disciplines and finally take a human centered approach are the salient underlying features of design thinking [5-7]. With the passage of time, along with food fortifications with vitamins and minerals, plant secondary metabolites, the polyphenolics, carotenoids are being impregnated in the designer nutraceuticals. Novel processing technologies has a significant impact on the quality attributes and effectivity of the bioactive especially during storage and transportations.

Thermal treatment often may deteriorate the effectivity of the active thermo-labile ingredients in food, thus compromising the quality. Consumers prefer fresh and minimally 
processed foods from the perception that processing of foods often leads to loss of its nutrient qualities. The main role of novel technologies in food processing is the extension of shelf life and maintaining the safety of fresh foods without altering the organoleptic and neurotherapeutic potentials. Along with improved thermal and non-thermal food processing techniques, the encapsulation technologies, recycle and reuse of nutrients, powder-based food products etc. offer the new state of art in food processing domain $[8,9]$. Generation of shock waves based on pulses of mechanical pressure or electrical discharges under water can be applied to tenderize meat cuts. This is due to the mechanical stress generated due to differences in acoustic properties to water and thus disrupting the structure of food matrix. These shock waves due to differences in acoustic properties also helps in enhanced extraction of bioactive from plant materials. In case of concentration of fruit juices, milk or dairy products use of techniques involving heat, vacuum or pressure may reduce the quality and involve a lot of energy. Use of non-thermal forward osmosis technique helps in concentration of beverages keeping quality attributes and proteins, vitamins, flavors and aromas intact. Sound waves at megahertz scale i.e. mega ultrasound technology can be applied in defoaming, texturization of foods and extraction of bioproducts. Dried snack items are all time favorite amongst the consumer world.

Ultrasound based drying technology helps in drying of food item at low temperature using sound waves with the simultaneous retention of thermo labile antioxidants and other nutritional components [10]. Flavor or aroma is an important quality attribute of food items. However, flavor quality often deteriorates during pasteurization or other thermal processing techniques. Thus, flavor management and flavor recovery system are an important aspect in food processing; spinning cone distillation columns are found to be more effective in flavor recovery than the conventional distillation systems [11]. Designing mass customized food basing on consumer categories viz. child, mothers, athletes, geriatric population with necessary value addition, tailored structural and chemical characteristics with requisite extended shelf life has raised the need of novel technologies [12]. 3-dimensional printing (3DP), is really an industrial revolution; consists of a raft of technologies with great translational potentials and wide applicability in biomedical research, reconstructive surgery, prosthetics, drug delivery research and food engineering [13]. 3-DP covers a wide group of additive manufacturing (AM) technologies viz. fused deposition modeling (FDM), selective laser sintering (SLS), micro extrusion, laser assisted bioprinting etc. 3D food printing is a developing huge market potential with a customized approach tailoring individualistic needs and thus a significant role in personalized nutrition. Geometric complexity, extended shelf life and mass customization enhancing organoleptic acceptability and maintaining neurotherapeutic potentials are some of the key areas providing an edge to 3D-food printing over other techniques [13-17]. The main purpose of 3DP technology is to develop 3-dimensional physical objects based on a computer model created in the program for graphic engineering in the form of .STL files.
3DP uses different materials like plastics, resins, polymers, metals, composites etc. however in 3D food printing the dough consists of edible food materials e.g. chocolate, essential food ingredients, synergistic value additive combinations keeping in mind their intrinsic properties and binding mechanisms during deposition of layers [12,15]. Concise discussions have been made on different thermal and non-thermal techniques with a focus on 3D food printing for mass customized diet and personalized nutrition.

\section{Thermal and non-thermal techniques in food processing}

The choice of technologies in food processing and food preservation is a crucial matter as food may contain thermo labile biologically active ingredients; often microbial infestation during shelf life making food safety questionable. Maintenance of organoleptic acceptability and nutritional value are both of vital concern while choosing food processing or preservation techniques. Non thermal food processing and preservation techniques are of much interest amongst food engineers and packaging scientists as the methods involve less energy expenditure with minimal impacts on food sensory and nutritional attributes and extension of shelf life by either killing or inhibiting microorganisms [18-20].

Amongst some of the novel thermal techniques, radio frequency heating is contact less heating with high penetrating power and good moisture leveling effect, often can be combined with conventional procedures and with less adverse effects on sensory and nutritional quality of foods. The technique is commercialized for processing nuts, seeds, spices, dry foods, defrosting fish and meats, blanching vegetables, drying of confectionaries and bakery products in post baking stages, vacuum drying of temperature sensitive products etc. However, equipment and production cost, unsuitability for fresh product treatment and probiotic fortified food treatment are some of its associated limitations [2,20].

Microwave heating generates heat within the products and thus offers food safety for the consumers, saves time, can be combined with conventional heating procedures with reduced carbon footprint. Apart from cooking, baking, pasteurizing, vacuum drying, puffing of snacks, reheating of previously cooked foods, defrosting of fish, meat and frozen foods, blanching, microwave assisted sterilization and pasteurization are some of the commercial and domestic applications. However high energy expenditure and expertise knowledge to understand uneven heating or thermal runaway are some of the limitations [2,20]. Infrared heating technique provides a uniform drying temperature with a high degree of process control, advantages of fast heating rate, shorter response time, possibility of selective heating and can be combined with conventional convective heating. This thermal procedure suffers from low penetration power, radiation energy may get absorbed at the food surface due to water content, and prolonged exposure of biological materials to infra-red may cause distortion. Infra-red heating finds applications in the drying of the fruits, vegetables, fish flakes, pasta, cereal grains, beans and nuts and also utilized in broiling, cooking, frying, roasting, heating etc. [2,20]. Amongst non thermal technologies used for preservation in food processing 
high pressure processing (pressures between 200 to $400 \mathrm{MPa}$ and temperature $<50{ }^{\circ} \mathrm{C}$ ) is found to preserve sensory attributes and nutritional qualities with uniform treatment and in less processing time. The method can be applied in packages and has not yet shown any evidences of toxicity. It is used for the preservation of sauces, pickles, yoghurt, salad dressings, meat, fruits and vegetables.

The method almost eliminates the use of chemical preservatives and kills bacteria and spores at higher temperature. For the purpose of preservation, the method causes permeabilization of cell membrane of the microbes. However, the treated food items must have lower water content and some food enzymes may be affected; the equipment is expensive and awaits some regulatory issues [1820]. Pulsed electric field processing (applied electric field intensity in range $20-80 \mathrm{kV} \mathrm{cm}^{-1}$, few pulses needed and treatment time less than $1 \mathrm{~s}$ ) is mostly used for the preservation of liquid food items (e.g. fruit juice, soups, liquid egg, milk) in a shorter processing time, maintaining sensory attributes and no adversities on food enzymes. The preservative action is due to the disruption of the microbial cell membrane due to electrical break down and electroporation effect. But it is difficult to use the method with conductive materials and often by products of electrolysis can cause adverse effects [18-20].

Cold plasma technique mostly used for decontamination of packaged foods, bottled beverages, fresh cut vegetables and meat; technically the method applies barrier glow discharge between two parallel electrodes where gas pressure, flow, frequency, composition, power of plasma excitation are the treatment conditions. It causes decontamination by destroying the lipid bilayer of the microbial cell membrane. However, lack of commercial instruments, scale up challenges, stability for large scale commercial operations and interactions of the electronically excited molecules with the food or packaging materials are the matters to be resolved [18-20]. Ultrasound processing with frequencies higher than $100 \mathrm{kHz}$ at intensities below $1 \mathrm{~W} \mathrm{~cm}^{-2}$ or frequencies between $18-100 \mathrm{kHz}$ at intensities higher than $1 \mathrm{~W} \mathrm{~cm}^{-2}$ (typically in the range of 10 $1000 \mathrm{~W} \mathrm{~cm}^{-2}$ ) is an effective tool for microbial inactivation, causing thinning of microbial cell membranes due to localized heating and production of free radicals.

The technique is used for preservation of fruit juices, in food emulsification, sterilization, extraction and freezing of fresh food stuffs. However, the method causes unwanted modifications of food structure and texture; sometimes negative modifications of sensory qualities, scale-up problems are some of the limitations to be addressed [18-20]. Irradiation procedure using 60 cobalt as the source of energy gamma ray and doses for sterilization 1-50kGy; it is successfully used for the preservation of fruits, vegetables, herbs, spices, meat and fish etc. Irradiation destroys the microbes by causing changes to chromosomal DNA or disruption in the cytoplasmic membrane. The method is reliable, energy efficient with excellent food penetrating power and minimal effect on sensory attributes and food quality. It is suitable for large scale production; however high capital costs, localized risk from irradiation, generation of free radicals are some of the associated problems [18-20].

\section{D food printing and mass customized food}

In this era where personalized nutrition and mass customized diet are gaining significance, 3DP food printing will help to develop designer foods with tailored shape, dimension, customized sensory attributes and personalized nutrition [17]. 3D food printing helps to design and develop food items with complex geometries, spatial solid structures, ornamental designs, different logos etc. [15]. The 3DP food printing methods include fused deposition manufacturing or hot melt extrusion, room temperature extrusion, selective laser sintering or hot air sintering, binder jetting, inkjet printing etc. Fused deposition manufacturing or hot melt extrusion applies material extruded from the nozzle layer by layer. The food material to be printed is heated to a certain temperature to obtain a semi molten state followed by quick and easy cooling on combination with the previous layer.

This technique is mostly used for 3D chocolate printing. In extrusion-based food printing firstly it is necessary to design a virtual 3D model. Then with the help of slicing software this virtual 3D model is translated into individual layer patterns and generating the machine code for printing (G-code generation). After uploading the codes into the printer, the next step is the choice of preferred food recipe and then the food printing starts. According to the layer patterns generated from the 3D model, the extruded material is dispensed either by moving the nozzle above the motorized stage or by moving the stage underneath the nozzle to form a layer. Each layer then binds with the previous layer on the stage to form the layer by layer 3D structure. Printed foods may undergo a post deposition cooking process like baking, frying etc. [15,21].

Extruder is the key component in the extrusion process of 3D food printing. The extruder mixes, heats, cools and shape highly viscous raw ingredients (both solid and liquid ingredients). Pre ground and conditioned food ingredients are fed into extruder and with the aid of mechanical or thermal energy are pressurized into viscoelastic fluid, texturized and shaped into die located at the end of the extruder and transited from high pressure to low pressure.

This digitally controlled robotic process builds layer by layer 3D food objects starting from loading with food ingredients, well controlled pushing of the ingredients out of the nozzle, moving the treated food material stream in a pre-defined path and eventually bonding the deposited layers the desired 3D food items [21]. In layer by layer 3D food printing, it is vital that layer can withstand enough strength to maintain its own weight as well as the weight of the other layers without significant deformation or change of shapes. Layers are often fused by spraying binder solution, laser heating or hot air treatment [15]. Selective sintering technology is based on melting together the particles of powders layer by layer. Powders rich in sugar content are very suitable for the procedure. Here computer-controlled laser irradiation is used for the purpose of sintering. A very thin layer of powder is first applied evenly on the bed and exposed to laser irradiation or hot air that melts and sinters the particles together. The substrate is lowered, and the same powder layer applied over, melted and sintered together as 
done in previous layer. The process is repeated till the total 3D printed food material is prepared. This technology is suitable for sugar and fat based materials with relatively low melting point. The Candy Fab applies a selective low velocity stream of hot air to sinter and melt a bed of sugar $[15,16]$. In powder bed jetting or binder jetting each powder layer evenly distributed across the fabrication platform and a binder solution sprayed to bind the consecutive powder layers. The powder material is stabilized through water mist to minimize disturbance caused by binder dispensing. The same procedure is repeated to form layer by layer complex food structures. The unbounded powder at each side is recovered for further reutilizations. Often sugar starch mixtures with flavored binders complex customized sculptural cakes etc. Though it's a fast fabrication procedure however high machine costs and rough surface finishing often are limitations $[15,16,21]$.

The Inkjet food printing technology dispenses stream or droplet from syringe-type print head in a drop-on demand. The ejected stream or droplets fall under gravity, impact on the substrate, and dry through solvent evaporation. The drops can form a two and half dimensional digital image as decoration or surface fill. This technology finds applications in printing 3D edible food products such as cookies, cakes, or pastries are created in a multilayered pattern. The De Good Innovations' Food Jet Printer uses pneumatic membrane nozzle-jets to deposit selected material drops onto pizza bases, biscuits and cupcakes [16]. Amongst the 3D printable food components include the natively printable materials e.g. chocolate, pasta dough, butter jelly, hydrogel, powdered starch or sugar that can be extruded directly from a syringe; traditional non printable materials e.g. rice, meat, fruits, vegetables etc. that are made printable or suitable for extrusion by adding hydrocolloids like xanthan gum or gelatin.

The third category are the alternative ingredients viz. insect powder acting as an immense protein source and replacing traditional meat [16]. Other than the availability of designer confectionary items, wide range of 3D chocolates, 3D food printing has taken a pivotal role in mass customized diet and personalized nutrition. Development of 3D fruit based snacks with further value additives like dried mushrooms, beans, citrus fruit juices containing requisite amount of vitamins, minerals, amino acids beneficial for children, mothers, athletes [17]; 3D printed smoothies which are blends of chosen fresh vegetables with fruit mixtures and other added ingredients like milk or honey are really considered as superfoods as they are natural foods with high health benefits [22]. Rapid advancements in 3D food printing has led to the development of customized fibrous meat with customized protein, fat, amino acid profile and sensory attributes [23]. Modernized food printers are using lasers and robotic arms to develop intricate decorative designs in food architecture.

The applicability of 3D printing in food technology is wide. This technology helps to achieve aesthetic and functional customization simultaneously. Apart from mass scale production and reduction of human labor, 3D food printing has a great impact on human health and combating of ailments. With a customized approach it satisfies both the purpose of personalized taste, nutritional requirements as per individualistic health needs. Novel food textures, extended shelf life, meal composition as per individualistic needs, creation of culinary state of art in our dishes, ease of transportation into most remote parts of the world or into space are the glimpses of the wide applicability of this technology. Above all bridging the food industry with the digital era is the groundbreaking application of this technology $[14-16,20]$.

\section{Conclusion}

In this era of personalized medicine, personalized nutrition and neurotherapeutic approach, the technologies associated with food engineering and food processing are also being revolutionized. Different thermal and non-thermal food processing technologies have their own advantages and limitations. However, to satisfy the global requirement; compared with manually customized food fabrication, 3D food printing does not require costly setup and hence is economical in small quantity production. The culinary intricate state of art in our food items depends on the process and planning rather than people's skill. The use of food printers on domestic scale may not be possible at this present juncture; but it has already attracted the global attention and may be possible in near future. However, 3D food printing is already the complementing technology in food processing. With automated customized food fabrication, the technology that has revolutionized the food industry today is sure to bring a versatile change in domestic kitchen in future. With the help of domestic scale 3D food printers customized diet or individualistic health need-based food can be printed in future.

\section{References}

1. Mitra A, Dewanjee D, Dey B (2012) Mechanistic studies of lifestyle interventions in Type 2 diabetes. World J Diabetes 3(12): 201-207.

2. Priyadarshini A, Rajauria G, Colm POD, Tiwari KB (2018) Emerging food processing technologies and factors impacting their industrial adoption. Crit Rev Food Sci Nutr, pp. 1-20.

3. Lewis DK, Freeman MBB (2010) The Role of Innovation and Technology in Meeting Individual Nutritional Needs. Journal of Nutrition 140(2): 426S-436S.

4. Anonymous (2019) Food tech and personalized nutrition- 1-From marketing to genetics. Paris Innovation review.

5. Olsen NV (2014) Design Thinking and Food Innovation. Proceedings in Food System Dynamics, pp. 135-143.

6. Hendrik NJS (2016) What design can bring to the food industry. International Journal of Food Design 1(2): 103-134.

7. Brodie EB, Telalbasic I (2017) Designing local food systems in everyday life through service design strategies. Design for Next: Proceedings of the $12^{\text {th }}$ European 20: 1-18.

8. Tokusoglu 0 (2018) Novel applications in nutrition and food science: Fortificated vitamins and polyphenols of innovative industrial foods and nutraceuticals. Journal of Food Health \& Technology Innovations 1(2).

9. Raso J (2017) Implementation of novel technologies. Taste of Science. $1-3$.

10. Tyers P (2019) Our top six new innovative food technologies. CSIRO Scope.

11. Pyle L (1994) Processed foods with natural flavour: The use of novel recovery technology. Nutrition \& Food Science 94(1): 12-14. 
12. Godoi FC, Prakash S, Bhandari BR (2016) 3d printing technologies applied for food design: status and prospects. Journal of Food Engineering 179: 44-54.

13. Katakam P, Dey B, Assaleh HF, Hwisa NT, Adiki KS, et al. (2015) Topdown and bottom-up approaches in $3 \mathrm{~d}$ printing technologies for drug delivery challenges. Crit Rev Ther Drug Carrier Syst 32(1): 61-87.

14. Singh P, Raghav A (2018) 3D Food Printing: A Revolution in Food Technology. Acta Scientific Nutritional Health 2(2): 11-12.

15. Izdebska J, Tryznowska ZZ (2016) 3D food printing-facts and future. Agro Food Industry Hi Tech 27(2): 33-37.

16. Sun J, Peng Z, Zhou W, Fuh JYH, Hong GS, et al. (2015) A review on 3d printing for customized food fabrication. Procedia Manufacturing 1: 308-319.

17. Derossi A, Caporizzi R, Azzollini D, Severini C (2018) Application of 3D printing for customized food. A case on the development of a fruit-based snack for children. Journal of Food Engineering 220: 65-75.

18. Jan A, Sood M, Sofi SA, Norzom T (2017) Non-thermal processing in food applications: A review. International Journal of Food Science and Nutrition 2(6): 171-180.
19. Hong ZZ, Wang LH, Zeng XA, Han Z, Brennan CS (2019) Non-thermal technologies and its current and future application in the food industry: a review. International Journal of Food Science and Technology 54(1): $1-13$.

20. Neetoo H, Chen H (2014) Alternative food processing technologies. food processing: principles and applications. In: Stephanie C (Eds.), $2^{\text {nd }}$ edn, John Wiley \& Sons, New Jersey, USA, pp. 137-169.

21. Sun J, Zhou W, Yan L, Huang D, Lin L (2018) Extrusion-based food printing for digitalized food design and nutrition control. Journal of Food Engineering 220: 1-11.

22. Severini C, Derossi A, Ricci I, Caporizzi R, Fiore A (2018) Printing a blend of fruit and vegetables. New advances on critical variables and shelf life of 3D edible objects. Journal of Food Engineering 220: 89-100.

23. Liu C, Ho C, Wang J (2017) The development of 3D food printer for printing fibrous meat materials. IOP Conf Series: Materials Science and Engineering 284: 012019. 